\title{
Differences in Neurodegeneration Between Kainic Acid-Injected GAERS and Wistar Rats
}

\author{
Umit S. SEHIRLI ${ }^{1,3}$, Ozlem KIRAZLI ${ }^{1}$, Kutluhan TEZCAN² ${ }^{2}$, Eren SAKALLI ${ }^{3}$, Filiz ONAT ${ }^{2,3}$ \\ ${ }^{1}$ Marmara University School of Medicine, Department of Anatomy, Istanbul, Turkey \\ ${ }^{2}$ Marmara University School of Medicine, Department of Pharmacology, Istanbul, Turkey \\ ${ }^{3}$ Eastern Mediterranean University School of Medicine, Famagusta, North Cyprus via Mersin 10, Turkey \\ Presented at the $40^{\text {th }}$ Meeting of the Society for Neuroscience, Chicago IL, USA, 17-21 October 2009.
}

Corresponding author: Umit S. SEHIRLI usehirli@marmara.edu.tr

\section{ABSTRACT}

AIM: To compare neurodegenerative changes using the Fluoro-Jade B staining, following status epilepticus induced by intraamygdaloid injection of kainic acid in Genetic Absence Epilepsy Rats from Strasbourg (GAERS) and non-epileptic control Wistar rats.

MATERIAL and METHODS: A single unilateral intra-amygdaloid kainic acid (750 ng) was administered in adult male GAERS and Wistar rats to induce status epilepticus. We recorded electroencephalogram (EEG) and behavioral changes throughout the experiments. After 1 week of the kainic acid injection, rats were sacrificed, and the brains were removed. We obtained 20 $\mu m$ sections and processed them for Fluoro-Jade B and Nissl staining, which were evaluated semi-quantitatively.

RESULTS: Following kainic acid injections, status epilepticus developed in all rats. In GAERS rats, motor seizures were considerably delayed, with no statistically significant difference in the number of seizures. However, statistically significant differences were observed in the Fluoro-Jade B staining in GAERS rats between contralateral and ipsilateral sides of the CA3, CA1, somatosensory cortex, entorhinal cortex, piriform cortex, reticular nucleus, putamen, and claustrum. In Wistar rats, the CA3, CA1, somatosensory cortex, entorhinal cortex, piriform cortex, reticular nucleus, amygdala, and laterodorsal nucleus exhibited significant differences. Comparing GAERS and Wistar rats, a statistically significant difference was observed for both sides of CA1. In both groups, the staining was prominent ipsilaterally, except for the claustrum in GAERS rats. However, the motor cortex remained unaffected in both groups. Neurodegenerative changes were not associated with the severity of seizures in both groups following the intra-amygdaloid kainic acid administration.

CONCLUSION: This study demonstrates that CA1 is the only region exhibiting a statistically significant difference between Wistar and GAERS rats.

KEYWORDS: Genetic absence epilepsy rats, Flouro-Jade B, Status epilepticus, Amygdala, CA3, Neuroscience, Absence epilepsy, Kainic acid, GAERS

\section{INTRODUCTION}

A bsence epilepsy, a form of generalized epilepsy, serves as a model for an approach to investigate epilepsies (27). However, the underlying mechanisms of absence epilepsy and its seizures remain partially defined. One accepted model for absence epilepsy is the Genetic Absence Epilepsy Rats from Strasbourg (GAERS), a well-validated model of absence epilepsy in humans $(11,13,24)$. In GAERS, all animals express genetically determined spontaneous spike-and-wave discharges (SWDs) on a cortical electroencephalogram (EEG), concurrent with behavioral arrest. The highly interconnected 
circuitry of the cortex and thalamus is known to play a vital role in the pathophysiology of absence epilepsy seizures. Studies have reported that bilateral SWDs are recorded in the frontoparietal and sensorimotor cortex and the posterolateral thalamic relay nuclei $(11,34)$. However, it has long been considered that limbic structures are not involved in absence seizures, as SWDs could not be recorded in the hippocampus and amygdala simultaneously with cortical SWDs in GAERS.

One of the enigmatic issues in GAERS is that these rats resist or experience a delay of secondary generalization of limbic seizures evoked by kindling. In addition, these rats resist or express a delayed response to intra-amygdaloid kainic acidinduced status epilepticus, which is a model of temporal lobe epilepsy $(1,3,7,16,26)$. These findings indicate an interaction between the limbic circuits triggered by kainic acid-induced status epilepticus or kindling and corticothalamic networks in GAERS. The administration of intra-amygdaloid kainic acid in non-epileptic Wistar rats induces status epilepticus, which is defined as a condition in which an uninterrupted seizure or several epileptic seizures occur recurrently for a minimum period of 1 hour; this period of seizures initially results in epileptogenesis followed by a chronic epileptic state $(9,20,22,28)$. In Wistar rats, kainic acid-induced status epilepticus is typically related to neurodegeneration and cell death throughout the brain. In addition, some studies have also reported observing selective degeneration of interneurons in the hilus of the dentate gyrus (DG) and pyramidal neurons in CA1 and CA3 $(9,25)$. Furthermore, the degeneration has also been observed in the amygdala, thalamus, olfactory cortex, neocortex, and substantia nigra $(17,31)$.

This study aims to ascertain any differences in the neurodegenerative changes between GAERS and non-epileptic Wistar rats, following intra-amygdaloid kainic acid-induced status epilepticus.

\section{- MATERIALS and METHODS}

We used male adult GAERS and Wistar control rats ( $>4$ months old) weighing 250-350 $\mathrm{g}$ for the experiments. All animals were housed in individual cages in air-conditioned rooms at $21^{\circ} \mathrm{C}$ on a $12 \mathrm{~h}$ light/dark cycle (light out from 08:00 to 20:00) with unlimited access to food and water. The protocol of experiments was approved by the Marmara University Ethics Committee for Experimental Animals (66.2004.Mar.).

The experiment was conducted in six groups as follows: (a) naive groups of GAERS and Wistar rats; (b) sham control groups of GAERS and Wistar rats; (c) kainic acid-injected GAERS and Wistar rats. In the kainic acid-injected groups, GAERS $(n=7)$ and Wistar $(n=7)$ rats were anesthetized with ketamine [100 mg/kg intraperitoneally (i.p.)] and xylazine [(10 $\mathrm{mg} / \mathrm{kg}$, (i.p.)]. The depth of anesthesia was confirmed with the absence of corneal and toe pinch reflexes. Next, the surgical area was cleaned with an antiseptic solution, and the hair were removed. The head of rats was placed into a stereotaxic instrument (Stoelting Model 51600; Stoelting Co., IL). Then, a longitudinal incision was made, and the periost was removed mechanically, followed by implanting two stainless steel screws with isolated wires on the right frontal and parietal cortex and free endings of wires soldered to a micro-connector for cortical EEG recordings. After that, a guide cannula (C312G; Plastics One, Roanoke, VI) was stereotaxically implanted into the right basolateral amygdala [coordinates: AP, $-2.6 \mathrm{~mm}$; ML, \pm 4.8 ; V, $-7.5 \mathrm{~mm}$ from the bregma according to the rat brain atlas of Paxinos and Watson (1998)]. Finally, the screw electrodes and cannula were fixed with cold dental acrylic. Postoperatively, rats were given $3 \mathrm{~mL}$ of isotonic salt solution subcutaneously to prevent fluid loss and were allowed to recover for 1 week.

After the recovery period, all rats were placed in Plexiglas cages. Using a BioAmp ML 136/PowerLab 8S System running Chart v.5 (ADI Instruments, UK), 1 hour baseline EEG was recorded before the injection in all experimental groups. In addition, EEG was recorded and evaluated in the GAERS groups to determine the basal SWD intensity. We defined SWD as a characteristic train of sharp asymmetric large-amplitude spikes and slow waves lasting at least 1 second (2). We used the first and last spikes as the start and end points of the SWDs. The cumulative duration and number of basal SWDs were analyzed over 1 hour. After that, we placed the internal cannula into the guide cannula, and all animals received 750 ng kainic acid in $300 \mathrm{~nL}$ of isotonic salt solution. Notably, injections were provided using a Hamilton syringe at 1 minute duration. After injections, the internal cannulas were left in the guide cannula for $5 \mathrm{~min}$. We recorded the behavioral and EEG changes until $2 \mathrm{~h}$ after the first observed motor seizure. Furthermore, behavioral changes after kainic acid injections were assessed per the following six-stage scale (32): stage 1, starting with mouth clonus; stage 2, automatisms (scratching and wet-dog-shake); stage 3, unilateral forelimb clonus; stage 4 , bilateral forelimb clonus; stage 5 , bilateral forelimb clonus with rearing and falling; and stage 6 , generalized tonic-clonic seizures.While stages 1 and 2 seizures were considered nonconvulsive limbic seizures, stages 3-6 were convulsive motor seizures.

During the experiments, we recorded time to the first limbic seizure, time to the first motor seizure, and the number of motor seizures. The seizures were terminated with $8 \mathrm{mg} / \mathrm{kg}$ i.p. diazepam at 2 hours after the first motor seizure. Rats were given $5 \mathrm{~mL}$ of isotonic salt solution subcutaneously to compensate for fluid loss and housed in individual cages for another week before decapitation.

In the naive groups, both male GAERS $(n=3)$ and Wistar $(n=$ 3) adult rats were only transcardially perfused as described earlier. We performed no stereotaxic procedure. In the sham control group, both male GAERS $(n=3)$ and Wistar $(n=3)$ adult rats were used. A cannula was implanted into the right basolateral amygdala (coordinates: $\mathrm{AP},-2.6 \mathrm{~mm}$; $\mathrm{ML}, \pm 4.8$; $\mathrm{V}$, $-7.5 \mathrm{~mm}$ from the bregma). After 1 week of stereotaxic surgery, $300 \mathrm{~nL}$ of artificial CSF was applied instead of kainic acid.

After 1 week of the status epilepticus period, we perfused rats transcardially with $4 \%$ paraformaldehyde solution and removed the brains, which were left in $4 \%$ paraformaldehyde solution overnight and then in a $30 \%$ sucrose solution for 72 hours. Then, $20 \mu \mathrm{m}$-thick slices were obtained on gelatincoated glass slides with a cryostat, and consecutive sections 
were obtained on different slides. While one group was stained with Fluoro-Jade B, the other was stained with Nissl staining.

\section{Fluoro-Jade B Staining Procedure}

The slides were dried at $37^{\circ} \mathrm{C}$ for at least $20 \mathrm{~min}$ and immersed in $100 \%$ ethyl alcohol for 3 minutes followed by $70 \%$ alcohol for 1 minute with a 1 minute change in distilled water. Then, the slides were transferred to a $0.06 \%$ potassium permanganate solution for 15 minutes, followed by 1 minute rinsing in distilled water and transfer to the Fluoro-Jade B staining solution for 30 minutes. In addition, a $0.01 \%$ stock solution of the dye was prepared by dissolving $10 \mathrm{mg}$ of Fluoro-Jade B in $100 \mathrm{~mL}$ of distilled water. The $0.001 \%$ working solution of Fluoro-Jade $\mathrm{B}$ was prepared by adding $10 \mathrm{~mL}$ of the stock Fluoro-Jade B solution to $90 \mathrm{~mL}$ of $0.1 \%$ acetic acid in distilled water. After staining, the sections were rinsed thrice for 1 minute each in distilled water. Finally, the excess water was drained off, and the slides were rapidly air dried (29).

\section{Data Analysis}

\section{EEG and Seizure Analysis}

The results are presented as mean \pm SEM. Using the Student's $t$-test, we analyzed the difference between the first motor seizures and the number of motor seizures after kainic acid injection in the Wistar and GAERS groups. We considered $p<$ 0.05 as statistically significant.

\section{Fluoro-Jade B Staining Evaluation}

The stained neurons were assessed semiquantitatively. If the number of neurons in a nucleus was between 1 and 10, it was accepted as (+); if 10 to 30 , it was accepted as (++); and if $>30$, it was accepted as $(+++)$. If there were no stained neurons, it was accepted as (-). We performed the statistical analysis with Graph Prism 5.0. Furthermore, the Mann-Whitney $U$-test was performed between groups with $\mathrm{p}<0.05$ as statistically significant.

\section{RESULTS}

\section{EEG Findings}

The baseline EEG recordings of all Wistar rats demonstrated no abnormal discharges. In GAERS rats, the 30 minutes cumulative duration of SWDs in the pre-injection period was $580.3 \pm 81.3$ seconds. Following kainic acid injections, all animals exhibited generalized convulsive seizures (Figure 1). Although the number of motor convulsive seizures did not attain statistical significance $(15.1 \pm 3.1$ for GAERS rats and $24.4 \pm 4.5$ for Wistar rats in a 2 hours period following kainic acid injections), the onset of motor seizures was significantly delayed in the GAERS groups $(65.1 \pm 9$ minutes for GAERS rats and $34.8 \pm 4.4$ minutes for Wistar rats; $p<0.05$; Figure 2A, B).

\section{Microscopic Evaluation}

Both Fluoro-Jade B- and Nissl-stained sections were assessed microscopically. The neurodegenerative findings were parallel for both Nissl and Fluoro-Jade B staining (Figures 3A, B; 4A, $B)$. Table I lists the sites stained by Fluoro-Jade B. In addition, we evaluated the differences between the ipsilateral and contralateral sides in each group and between the GAERS and Wistar rats in naïve, sham control, and kainic acid-injected groups. Notably, we found no Fluoro-Jade B-labeled cells in the naïve groups. Minimal Fluoro-Jade B-labeled cells were present in the sham control Wistar and GAERS animals in the cannula trajectory.

We compared the ipsilateral and contralateral sides of kainic acid-injected Wistar and GAERS groups, as well as the

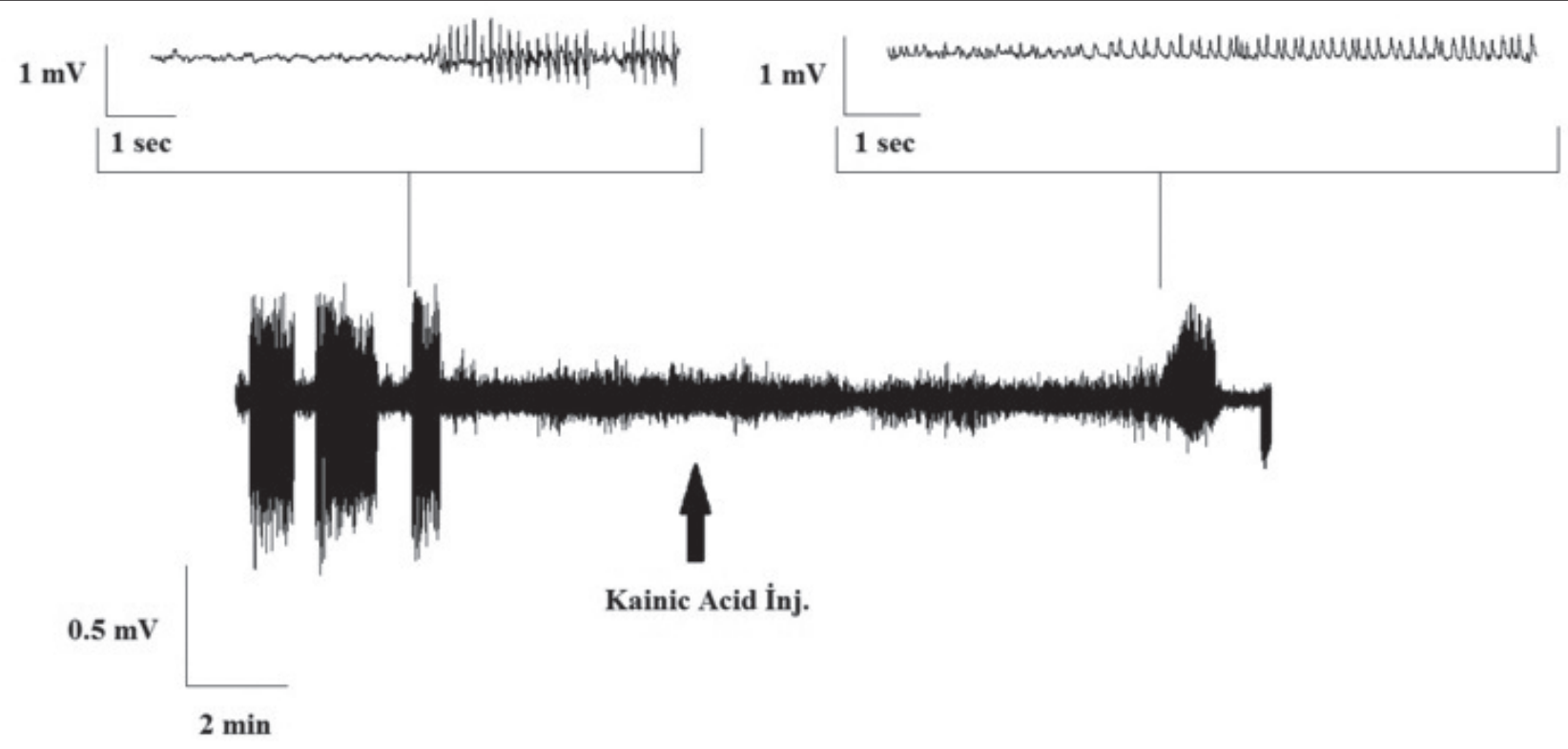

Figure 1: EEG trace of GAERS shows the suppression of SWD's after kainic acid injection (black arrow) and the emergence of kainic acid-induced discharges. Detailed traces for a SWD (top left) and for kainic acid-induced discharges (top right). 

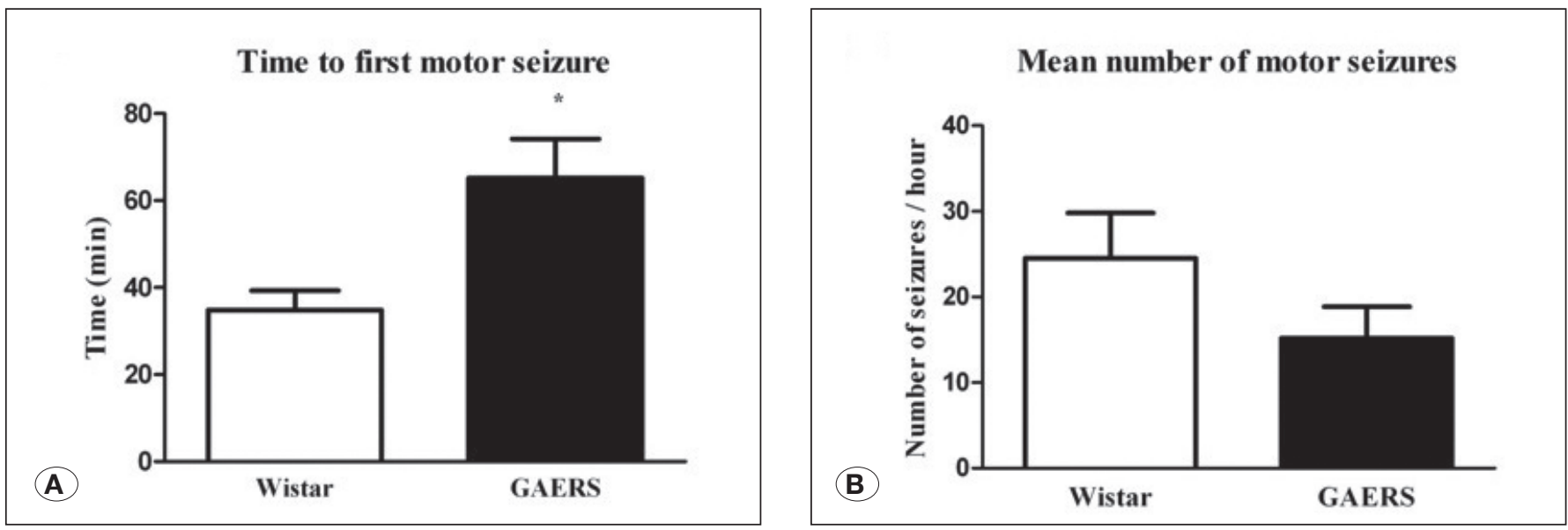

Figure 2: A) Time to the first motor seizures after kainic acid injection, Wistar rats $(n=7)$ and GAERS $(n=7) .{ }^{*}, p<0.05$, the first motor seizure was significantly delayed in the GAERS group compared with the Wistar group. B) the number of motor seizures after kainic acid injection in Wistar rats $(n=7)$ and GAERS $(n=7)$.
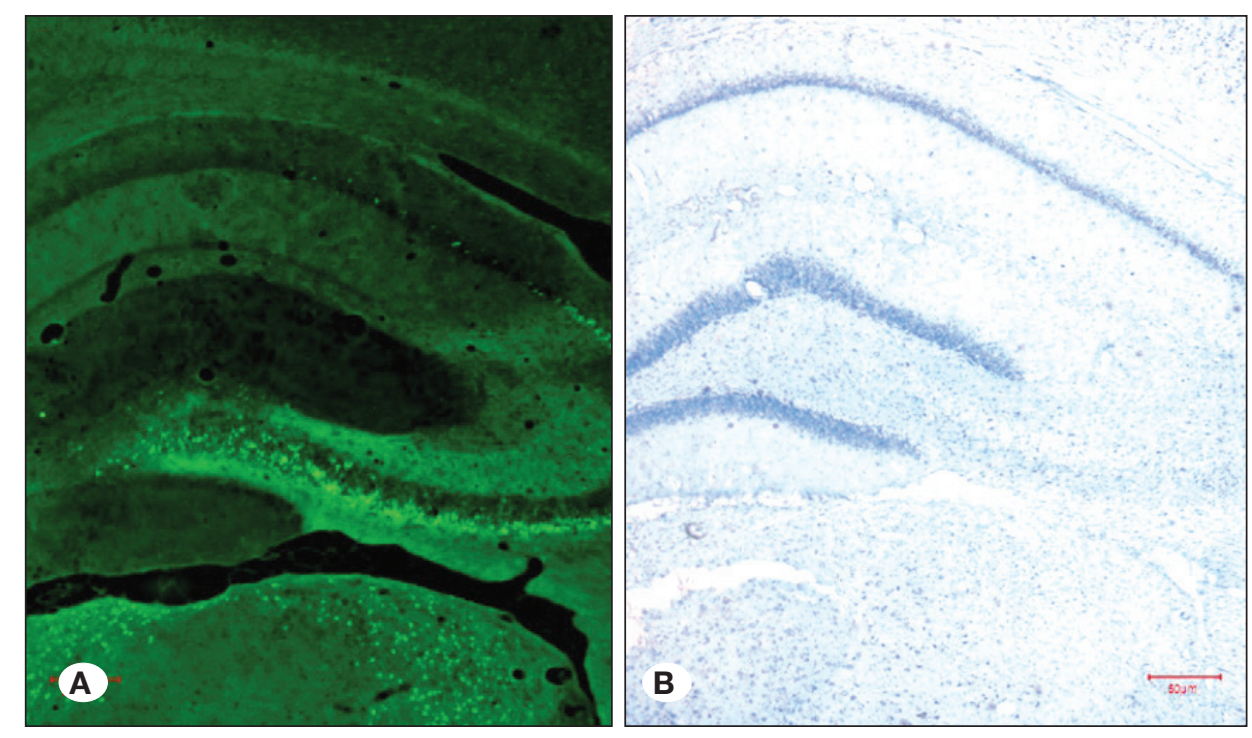

Figure 3: A) The Fluoro-Jade B staining of the hippocampus of intra-amygdaloid kainic acid-injected Wistar rat. B) The Nissl staining of consecutive sections.
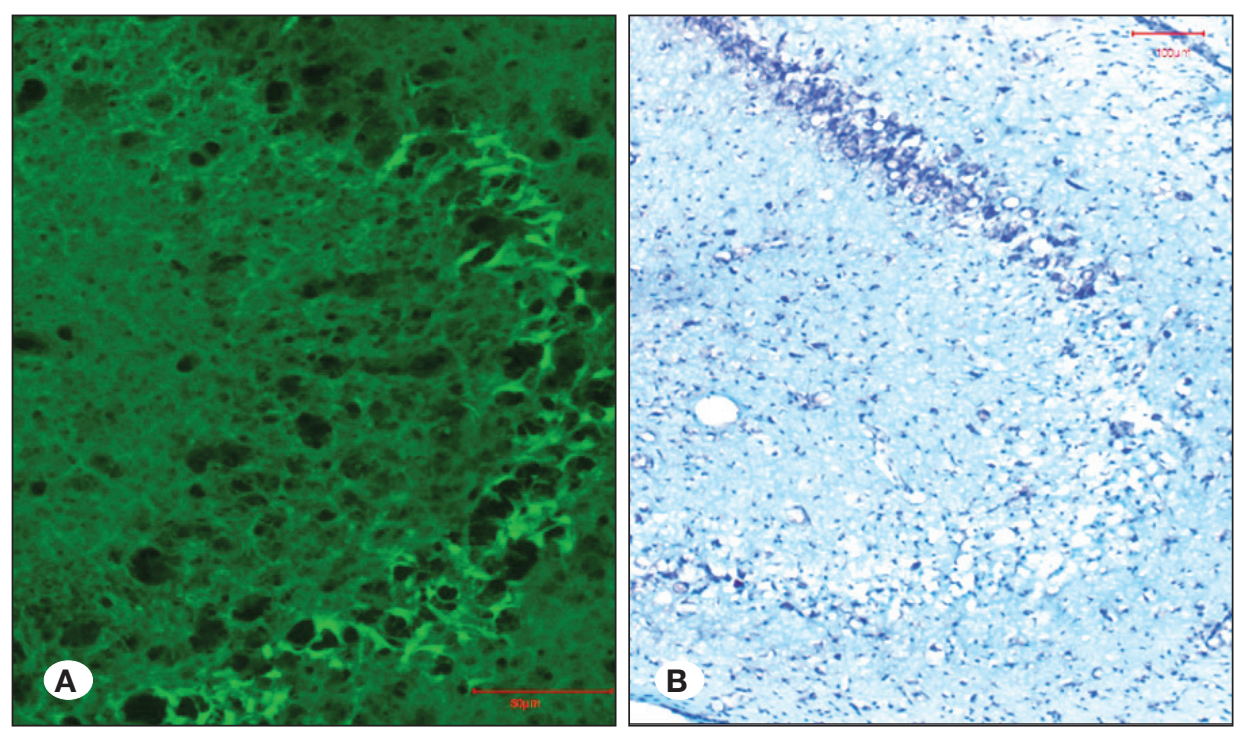

Figure 4: A) The Fluoro-Jade B staining of the CA3 of intra-amygdaloid kainic acid-injected GAERS. B) The NissI staining of consecutive sections. 
Table I: Fluoro-Jade B Stained Areas

\begin{tabular}{|c|c|c|c|c|}
\hline Kainic acid injected rats & \multicolumn{2}{|c|}{ GAERS } & \multicolumn{2}{|c|}{ Wistar } \\
\hline \multicolumn{5}{|l|}{ Hippocampus } \\
\hline CA1 & +++ & + & + & -- \\
\hline CA3 & +++ & -- & + & -- \\
\hline Motor cortex & -- & -- & -- & -- \\
\hline Somatosensorial cortex & ++ & + & ++ & -- \\
\hline Enthorinal cortex & ++ & + & ++ & + \\
\hline Piriform & ++ & + & ++ & + \\
\hline Mediodorsal thalamic nuc. & ++ & ++ & ++ & ++ \\
\hline Lateradorsal thalamic nuc. & ++ & ++ & ++ & -- \\
\hline Reticular thalamic nuc. & + & -- & ++ & -- \\
\hline \multicolumn{5}{|l|}{ Other Structures } \\
\hline Lateral septal nuc. & ++ & ++ & ++ & ++ \\
\hline Amygdala & ++ & + & ++ & + \\
\hline Putamen & ++ & -- & ++ & -- \\
\hline Claustrum & + & ++ & + & ++ \\
\hline
\end{tabular}

ipsilateral and contralateral sides. In GAERS groups, we observed statistically significant differences in the FluoroJadeB staining between the contralateral and ipsilateral sides of the CA1, CA3, reticular cortex, entorhinal cortex, piriform cortex, somatosensory cortex, putamen, and claustrum. In Wistar rats, the CA1, CA3, somatosensory cortex, entorhinal cortex, piriform cortex, reticular nucleus, amygdala, and the laterodorsal nucleus exhibited significant differences (Figure $5 A-J)$. In this study, another crucial finding was the unaffected motor cortex in both groups.

Comparison of GAERS and Wistar rats revealed statistically significant differences between both groups in both sides of the CA1 region of the hippocampus, somatosensory cortex, and laterodorsal thalamic nucleus where GAERS rats had dense staining.

\section{DISCUSSION}

One of the major findings of this study was the delay in the first convulsive seizure after administering kainic acid in the GAERS rats compared with the Wistar rats; this finding corroborated our prior work (18). These studies suggest that although GAERS rats exhibited convulsive seizures and status epilepticus after the kainic acid administration, the time to the first convulsive seizure was considerably longer compared with the Wistar group. All these findings strengthen the hypothesis that GAERS rats exhibit a delay in the secondary generalization of focal limbic seizures. A study reported that the first seizure induced by low kainic acid doses was less severe and exhibited a longer latency in GAERS compared with non-epileptic controls (33).

Another finding of this study is the neurodegenerative changes because of status epilepticus after the kainic acid administration into the amygdala, which was observed in both GAERS and Wistar rats. In addition, extensive degeneration was observed in several brain regions in both groups. The neurodegeneration in the contralateral sides of the CA1, somatosensory cortex, and the laterodorsal thalamic nucleus in Wistar rats was spared; however, neurodegeneration was observed in these areas of the GAERS rats.

The somatosensory cortex, thalamic nuclei, and thalamic reticular nucleus are implicated in the pathogenesis of absence epilepsy $(4,35)$. In this study, the thalamic structures primarily affected were the reticular, laterodorsal, and mediodorsal nuclei in both kainic acid-injected Wistar and GAERS rats. In addition, the ipsilateral thalamic reticular nucleus exhibited degenerated neurons in both groups. However, in both groups, the contralateral sides of the thalamic reticular 

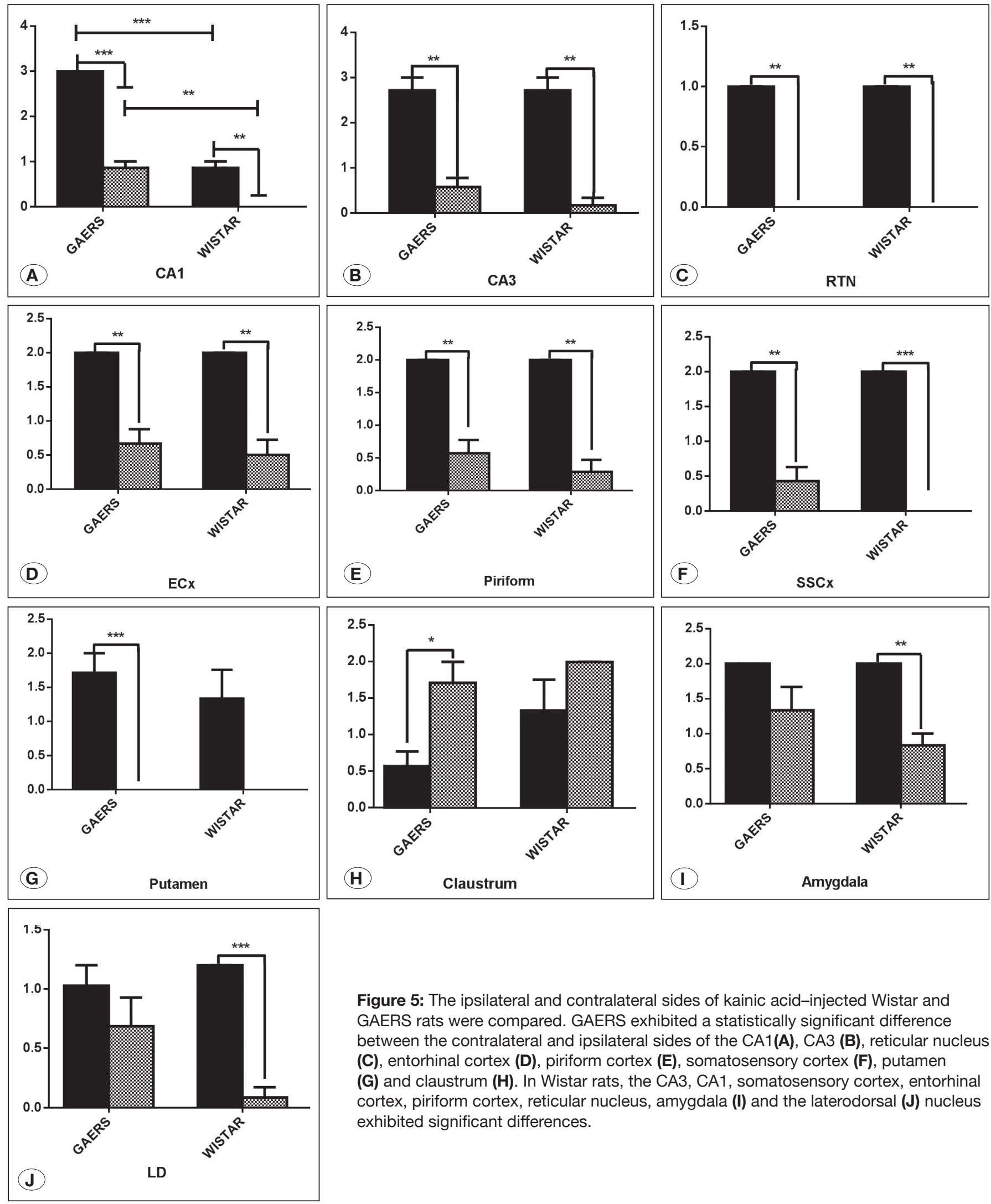

Figure 5: The ipsilateral and contralateral sides of kainic acid-injected Wistar and GAERS rats were compared. GAERS exhibited a statistically significant difference between the contralateral and ipsilateral sides of the CA1(A), CA3 (B), reticular nucleus (C), entorhinal cortex (D), piriform cortex (E), somatosensory cortex (F), putamen (G) and claustrum (H). In Wistar rats, the CA3, CA1, somatosensory cortex, entorhinal cortex, piriform cortex, reticular nucleus, amygdala (I) and the laterodorsal (J) nucleus exhibited significant differences. 
nucleus were spared compared with the ipsilateral sides. Recently, we reported that the thalamic reticular nucleus was associated with the resistance to the generalization of limbic seizures (8). Although the laterodorsal thalamic nucleus has not been considered as one of the thalamic nuclei closely associated with the epilepsy mechanism, progressive damage and altered $\mathrm{GABA}_{\mathrm{A}}$ receptor subunit expression in the laterodorsal thalamic nucleus have been reported in posttraumatic epilepsy in adult rats (14). These findings and our study suggest that the laterodorsal thalamic nucleus could be part of the epileptic network. Furthermore, in both groups, the mediodorsal thalamic nucleus was affected bilaterally; the role of the mediodorsal thalamic nucleus in epilepsy has been described previously $(5,21)$. A prior study reported that the mediodorsal thalamic nucleus plays a role in the propagation of a seizure because of its limbic connections (21). Moreover, the mediodorsal thalamic nucleus has been considered to play a role in absence seizures. Kato et al. reported that the kainic acid administration into the mediodorsal thalamic nucleus triggered cognitive impairment and considered this region as the mechanism of petit mal seizures (19). Bertram et al. reported that the mediodorsal thalamic nucleus plays a role in the limbic seizure circuit and the spread of seizures; the mediodorsal thalamic nucleus acts as a key control point of the seizure, which comes from limbic sites (6). Our findings corroborate the literature to elucidate the role of the mediodorsal thalamic nucleus in epilepsy; however, these findings do not attribute any specific role to the mediodorsal thalamic nucleus in the absence epilepsy mechanism.

In the hippocampus, a crucial difference was observed between the CA1 regions of the two groups, kainic acidinjected Wistar rats and kainic acid-injected GAERS rats. The contralateral side of Wistar rats was preserved, and the ipsilateral side was less affected compared with GAERS, suggesting a susceptibility of the CA1 region in GAERS rats to degeneration, but the configuration of this study could not elucidate the reason for this susceptibility. However, further studies are warranted to focus on the extensive degeneration of the CA1 in kainic acid-injected GAERS rats. We observed the degeneration on both sides of GAERS and Wistar rats at the dorsal endopiriform nucleus, piriform, entorhinal cortex, lateral septal nucleus, and amygdala. These structures are either part or closely associated with the limbic system. Furthermore, neonatal status epilepticus was reported to result in extensive neuronal death in the ipsilateral hippocampal CA1 and CA3 subfields and the hilus, as assessed by DNA fragmentation and Fluoro-Jade B staining after 72 hours (15). Previous studies suggested that the amygdala, thalamus, entorhinal cortex, and isocortex, especially the hippocampus, are the primary sites of neuron loss and tissue shrinkage in patients with temporal lobe epilepsy(10). Chen and Buckmaster reported that the systemic treatment of kainic acid causes volume loss in the amygdala and olfactory cortex (10). A recent study reported a correlation between neuron loss and motor seizures in the CA1, CA3, and hilum (23).

Degenerated neurons were observed in the putamen and claustrum; these structures are associated with the basa ganglia. Several lines of evidence suggest that the basal ganglia play a role in the modulation, propagation, and cessation of different types of experimental seizures, including kainic acid injection and absence epilepsy $(12,19,30)$. The contralateral sides of the CA3, reticular nucleus, and the putamen were spared in the Wistar and GAERS rats.

\section{CONCLUSION}

This study has demonstrated that the ipsilateral CA1, somatosensory cortex, and laterodorsal thalamic nucleus are affected in GAERS rats suggesting that these regions play a role in the thalamocortical pathway in absence epilepsy.

\section{ACKNOWLEDGMENTS}

We thank Prof. Rezzan Gulhan for contribution to the study.

This study was supported by Marmara University Research Fund SAG-E-300409-0087 and Brain Research Society Turkey.

\section{REFERENCES}

1. Aker RG, Yananli HR, Gurbanova AA, Ozkaynakci AE, Ates $\mathrm{N}$, van Luijtelaar G, Onat FY: Amygdala kindling in the WAG/ Rij rat model of absence epilepsy. Epilepsia 47(1):33-40, 2006

2. Akman O,Demiralp T, Ates N, OnatFY:Electroencephalographic differences between WAG/Rij and GAERS rat models of absence epilepsy. Epilepsy Res 89(2-3):185-193, 2010

3. Akman O, Karson A, Aker RG, Ates N, Onat FY: Hippocampal kindling in rats with absence epilepsy resembles amygdaloid kindling. Epilepsy Res 81(2-3):211-219, 2008

4. Avoli M, Gloor P: Interaction of cortex and thalamus in spike and wave discharges of feline generalized penicillin epilepsy. Exp Neurol 76(1):196-217, 2001

5. Bertram EH, Mangan PS, Zhang D, Scott, CA, Williamson JM: The midline thalamus: Alterations and a potential role in limbic epilepsy. Epilepsia 42(8):967-978, 1982

6. Bertram EH, Zhang D, Williamson JM: Multiple roles of midline dorsal thalamic nuclei in induction and spread of limbic seizures. Epilepsia 49(2):256-268, 2008

7. Carcak N, Aker RG, Ozdemir O, Demiralp T, Onat FY: The relationship between age-related development of spike-andwave discharges and the resistance to amygdaloid kindling in rats with genetic absence epilepsy. Neurobiol Dis 32(3):355363,2008

8. Carcak N, Zheng T, Ali I, Abdullah A, French C, Powell KL, O'Brien TJ: The effect of amygdala kindling on neuronal firing patterns in the lateral thalamus in the GAERS model of absence epilepsy. Epilepsia 55(5):654-665, 2014

9. Castro OW, Furtado MA, Tilelli CQ, Fernandes A, Pajolla GP, Garcia-Cairasco N: Comparative neuroanatomical and temporal characterization of Fluoro Jade-positive neurodegeneration after status epilepticus induced by systemic and intrahippocampal pilocarpine in Wistar rats. Brain Res 1374:43-55, 2011

10. Chen S, Buckmaster PS: Stereological analysis of forebrain regions in kainate-treated epileptic rats. Brain Res 1057(1-2): 141-152, 2005 
11. Danober L, Deransart C, Depaulis A, Vergnes M, Marescaux C: Pathophysiological mechanisms of genetic absence epilepsy in the rat. Prog Neurobiol 55(1):27-57, 1998

12. Depaulis A: The inhibitory control of the substantia nigra over generalized non-convulsive seizures in the rat. J Neural Transm Suppl 35:125-139, 1992

13. Depaulis AVLG: Genetic models of absence epilepsy in the rat. In: Pitkänen A, Schwartkroin PA, Moshé SL (ed), Models of Seizures and Epilepsy. Elsevier: Academic Press, 2005:233248

14. Drexel M, Puhakka N, Kirchmair E, Hortnagl H, Pitkanen A, Sperk G: Expression of GABA receptor subunits in the hippocampus and thalamus after experimental traumatic brain injury. Neuropharmacology 88:122-133, 2015

15. Dunleavy M, Shinoda S, Schindler C, Ewart C, Dolan R, Gobbo OL, Kerskens CM, Henshall DC: Experimental neonatal status epilepticus and the development of temporal lobe epilepsy with unilateral hippocampal sclerosis. Am J Pathol 176(1): 330-342, 2010

16. Eskazan E, Onat FY, Aker R, Oner G: Resistance to propagation of amygdaloid kindling seizures in rats with genetic absence epilepsy. Epilepsia 43(10):1115-1119, 2002

17. Fujikawa DG: The temporal evolution of neuronal damage from pilocarpine-induced status epilepticus. Brain Res 725(1): 11-22, 1996

18. Gurbanova AA, Aker RG, Sirvanci S, Demiralp T, Onat FY: Intra-amygdaloid injection of kainic acid in rats with genetic absence epilepsy: The relationship of typical absence epilepsy and temporal lobe epilepsy. J Neurosci 28(31):78287836, 2008

19. Kato K, Urino T, Hori T, Tsuda H, Yoshida K, Hashizume K, Tanaka T: Experimental petit mal-like seizure induced by microinjection of kainic acid into the unilateral mediodorsal nucleus of the thalamus. Neurol Med Chir (Tokyo) 48(7):285290; discussion 290-291, 2008

20. Kobow K, Auvin S, Jensen F, Loscher W, Mody I, Potschka H, Prince D, Sierra A, Simonato M, Pitkanen A, Rho JM: Finding a better drug for epilepsy: Antiepileptogenesis targets. Epilepsia 53(11):1868-1876, 2012

21. Kubova H, Druga R, Haugvicova R, Suchomelova L, Pitkanen A: Dynamic changes of status epilepticus-induced neuronal degeneration in the mediodorsal nucleus of the thalamus during postnatal development of the rat. Epilepsia 43 Suppl 5:54-60, 2002

22. Löscher W: Strategies for antiepileptogenesis: Antiepileptic drugs versus novel approaches evaluated in post-status epilepticus models of temporal lobe epilepsy In: Noebels JL, Avoli M, Rogawski MA, Olsen RW, Delgado-Escueta AV, (eds), Jasper's Basic Mechanisms of the Epilepsies, $4^{\text {th }}$ ed. Bethesda (MD): National Center for Biotechnology Information (US), 2012
23. Maia GH, Quesado JL, Soares JI, do Carmo JM, Andrade PA, Andrade JP, Lukoyanov NV: Loss of hippocampal neurons after kainate treatment correlates with behavioral deficits. PLoS One 9(1):e84722, 2014

24. Marescaux C, Vergnes M, Depaulis A: Genetic absence epilepsy in rats from Strasbourg-a review. J Neural Transm Suppl 35:37-69, 1992

25. Mohapel P, Ekdahl CT, Lindvall O: Status epilepticus severity influences the long-term outcome of neurogenesis in the adult dentate gyrus. Neurobiol Dis 15(2):196-205, 2004

26. Onat FY, Aker RG, Gurbanova AA, Ates N, van Luijtelaar G: The effect of generalized absence seizures on the progression of kindling in the rat. Epilepsia 48 Suppl 5:150-156, 2007

27. Onat FY, van Luijtelaar G, Nehlig A, Snead OC $3^{\text {rd: }}$ The involvement of limbic structures in typical and atypical absence epilepsy. Epilepsy Res 103(2-3):111-123, 2013

28. Pitkanen A, Sutula TP: Is epilepsy a progressive disorder? Prospects for new therapeutic approaches in temporal-lobe epilepsy. Lancet Neurol 1(3):173-181, 2002

29. Schmued LC, Hopkins KJ: Fluoro-Jade B: A high affinity fluorescent marker for the localization of neuronal degeneration. Brain Res 874(2):123-130, 2000

30. Slaght SJ, Paz T, Mahon S, Maurice N, Charpier S, Deniau JM: Functional organization of the circuits connecting the cerebral cortex and the basal ganglia: Implications for the role of the basal ganglia in epilepsy. Epileptic Disord 4 Suppl 3:S9-22, 2002

31. Turski L, Cavalheiro EA, Czuczwar SJ, Turski WA, Kleinrok Z: The seizures induced by pilocarpine: Behavioral, electroencephalographic and neuropathological studies in rodents. Pol J Pharmacol Pharm 39(5):545-555, 1987

32. Veliskova J, Moshe SL: Update on the role of substantia nigra pars reticulata in the regulation of seizures. Epilepsy Curr 6(3): 83-87, 2006

33. Vergnes M, Boehrer A, Reibel S, Simler S, Marescaux C: Selective susceptibility to inhibitors of GABA synthesis and antagonists of $\mathrm{GABA}(\mathrm{A})$ receptor in rats with genetic absence epilepsy. Exp Neurol 161(2):714-723, 2000

34. Vergnes M, Marescaux C, Depaulis A: Mapping of spontaneous spike and wave discharges in Wistar rats with genetic generalized non-convulsive epilepsy. Brain Res 523(1):87-91, 1990

35. Vergnes M, Marescaux C, Depaulis A, Micheletti G, Warter $\mathrm{JM}$ : Spontaneous spike and wave discharges in thalamus and cortex in a rat model of genetic petit mal-like seizures. Exp Neurol 96(1):127-136, 1987 